\title{
Software Architecture and Hardware Organization in Mu2e Solenoid Field Mapping System
}

\author{
J. M. Nogiec, P. Akella, S. Feher, J. Grudzinski, T. Strauss, R. L. Talaga, and L. Xia
}

\begin{abstract}
The Mu2e field mapping system is designed to produce high accuracy field maps of the detector solenoid used in the experiment. The data acquisition system is mobile and uses a selfpropelled mapper with rotating arms equipped with 3D Hall probes. The measurements require not only accurate magnetic field readouts, but also accurate locations of all Hall probes while measuring data. The latter is accomplished by using the laser tracker to measure positions of several retroreflectors on the field mapper during data acquisition. The measurement process requires scanning the whole space inside the large solenoid and takes many hours to complete, which necessitates its full automation. The automation software includes control of the mapper, readout of Hall and NMR probes and control of a laser tracker, including prediction of retroreflector positions and execution of quality control checks. The software architecture and data acquisition hardware of the field mapping system are described, with special attention given to control of the laser tracker and its integration with the rest of the system.
\end{abstract}

Index Terms-Magnetic field measurement, superconducting magnets, automatic testing, software architecture.

\section{INTRODUCTION}

$\mathbf{T}$ HE Muon-to-Electron Conversion Experiment (Mu2e) is under construction at Fermilab. This experiment will probe for indication of physics beyond the Standard Model by searching for evidence of charged lepton flavor violation [1] through the direct conversion of muons into electrons.

The Mu2e magnet system is the heart of the experiment. It consists of the Production solenoid (PS), the Transport Solenoid (TS), and the Detector Solenoid (DS). The PS receives a proton beam from the Fermilab accelerator complex, which is directed at a wolfram target. The particles emerging from the collision are directed downstream toward the TS. Inside the TS, absorbers and collimators are used to eliminate other particles beside $\mu^{-}$. In the DS, muons hit the aluminum stopping target, and a graded field collects conversion electrons and guides them toward the tracker and calorimeter detectors, which are installed in a uniform 1 Tesla field.

The magnetic field of the DS is mapped and validated with a calculated, model-based field map. To perform this mapping, a flexible Field Mapping System (FMS) is used, consisting of a specialized field mapping device, precisely calibrated Hall

This work was supported in part by the Fermi Research Alliance under DOE Contract DE-AC02-07CH11359. (Corresponding author: Jerzy Nogiec.)

J. Nogiec, P. Akella, S. Feher, T. Strauss are with Fermi National Accelerator Laboratory, Batavia, IL 60510 USA (e-mail: nogiec@fnal.gov). J. Grudzinski, R. Talaga, L. Xia are with Argonne National Laboratory, Argonne, IL 60439 USA. sensors, and a laser tracker to measure their positions and orientations.

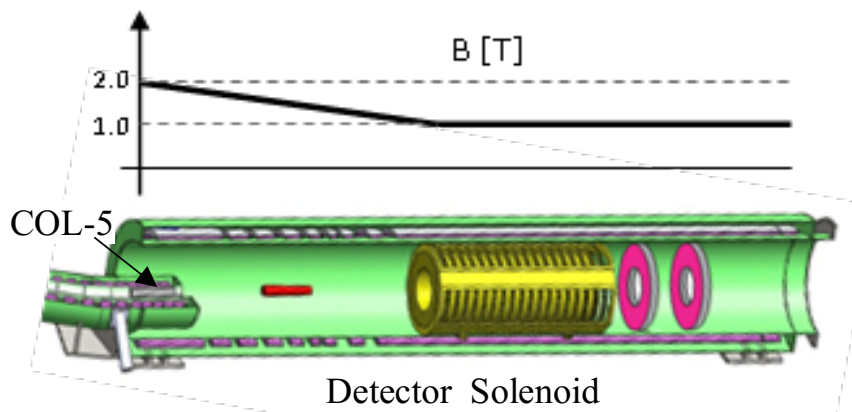

Fig. 1. Mu2e Detector Solenoid and its axial magnetic field.

\section{FIELD MAPPING REQUIREMENTS}

\section{A. Mu2e Detector Solenoid}

The DS is the last in the chain of Mu2e solenoids, with the last section of the TS (COL-5) protruding into its cryostat (Fig. 1). The diameters of the magnet cryostat are $2.656 \mathrm{~m}$ (outer) and $1.9 \mathrm{~m}$ (inner), and its length is $10.9 \mathrm{~m}$ [2]. It houses the aluminum muon stopping target, tracker, and calorimeter.

The magnetic flux density, B, along the DS axis is shown in Fig. 1. The DS needs to provide a graded magnetic field in the approximately three-meter-long section of the target (from $2 \mathrm{~T}$ to $1 \mathrm{~T}$ ) and a nearly constant magnetic field (1 T) in the spectrometer and calorimeter regions (ca. 6 meters). The graded field section collects conversion electrons and guides them toward the tracker and calorimeter detectors. The graded field region and the spectrometer region are separated by a roughly one-meter-long transition region.

\section{B. Field Mapping Accuracy}

The requirement for the field gradient in the target region is $-0.25 \pm 0.05 \mathrm{~T} / \mathrm{m}$. The maximum allowed field nonuniformity $(\Delta \mathrm{B} / \mathrm{B})$ is $0.01 \%$ for the spectrometer region and $0.05 \%$ for the calorimeter region.

The near-constant-field region of the DS spectrometer volume requires the highest level of measurement accuracy, 
$0.01 \%$ ( $1 \mathrm{G}$ in $1 \mathrm{~T}$ field). The direction of the $\mathrm{B}$ field vector should be known to better than $0.1 \mathrm{mrad}$ [3].

For comparison, the field mapping precision required for the tracking volume of the CMS detector was $0.07 \%$ [4]. The 3D Hall probes for the ATLAS detector were calibrated with $0.01 \%$ accuracy up to $1.4 \mathrm{~T}$ and $0.05 \%$ up to $2.5 \mathrm{~T}$, and the alignment accuracy was expected to be $\pm 2 \mathrm{mrad}$ for both calibrations [5].

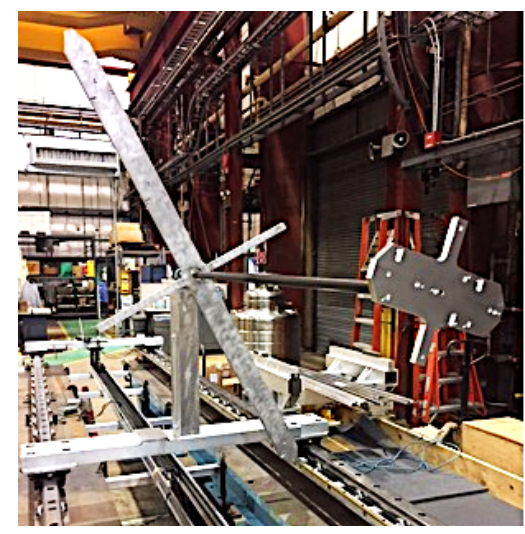

Fig. 2. Mu2e field mapper integration testing at ANL.

\section{Field Mapping Process}

The large volume of the DS necessitated the development of a self-propelled mapper device equipped with 3D Hall probes and an NMR probe (Fig. 2) The mapper traverses the full length of the DS magnet on built-in rail tracks to guide the detector assembly.

At each longitudinal position, several readouts are performed, each at a different angular position of the mapper's rotating arms. The positions of the arms are precisely determined by a laser tracker, which allows for calculating and precisely determining the Hall probe positions.

The field mapping process is completely automated. Each measurement includes readouts of all Hall and NMR sensors, Hall sensor temperatures, the positions of retroreflectors on the mapper arms and the current in the magnet. The measurement space is divided into multiple measurement regions, each with a different granularity of field mapping positions.

\section{FMS HARDWARE}

The data acquisition and control hardware consist of instruments and probes connected to the measurement computer via a private computer network and USB. USB is used to connect the Hall probes and NMR readout, and the other instruments use network interfaces.

\section{A. Field Mapper}

The mapper is self-propelled and similar in its concept to the ATLAS [5] and CMS [4] mappers, but different in that is uses ultrasonic motors for propulsion and measures volumes of two cylinders, with different diameters, on axis with the magnet.
The FMS mapper has two sets of arms, resembling small and large propellers, placed on a common rotating shaft and rotated together [3]. The arm spans are: 64.96 " and 30 " for the large "propeller", and 9.45" and 8.5" for the small "propeller". Both "propellers" will map the volume of the DS. The smaller "propeller" will also map the most downstream section of the TS (COL-5).

The main mechanical structure of the field mapper is made from aluminum as much as possible, but there are a few other materials used:

- The shaft between the two propellers is a carbon fiber tube.

- The reduction gears are stainless steel.

- The bearings for the propeller shaft are plastic.

- All the screws are brass.

Control of the mapper is accomplished using the Galil DMC-4040 motion controller, which uses an ultrasonic motor driver (Shinsei D6060E) to actuate the mapper's ultrasonic motors (Shinsei USR60-E3NT). Both the driver and the motors are mounted in the mapper and were therefore selected to be non-magnetic.

\section{B. Hall probes and their readout}

B-sensor probes are placed on the rotating arms of the mapper (Fig. 3). These are 3D Hall probes designed by CERN and produced by NIKHEF [6]. To improve their accuracy, a specialized system to calibrate them in stable temperatures was developed at Fermilab [7]. The B-sensor probe incorporates three Siemens KSY44 Hall sensors, a microcontroller, Cirrus Logic CS5524 24-bit, 4-channel ADC, and a multiplexor. The SPI signals (magnetic field values, and temperature) from all the probes are converted to CAN messages in the mBATCAN module and finally interfaced with the computer via USBCAN adapter (Kvaser USBcan II). The readout of all sensors occurs in sub-second time.

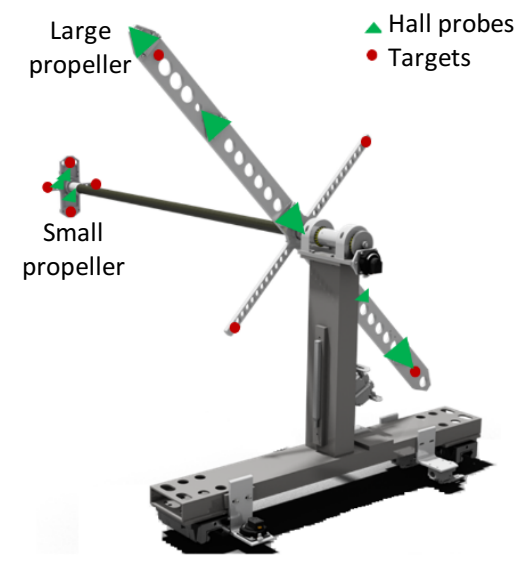

Fig. 3. Mu2e field mapper.

\section{NMR readout}

Metrolab PT2026 NMR Precision Teslameter [8] employs a pulsed-wave resonance detection and achieves accuracy of $+/$ 5 ppm, with search time of less than 1 second when using Hall probe assistance. 

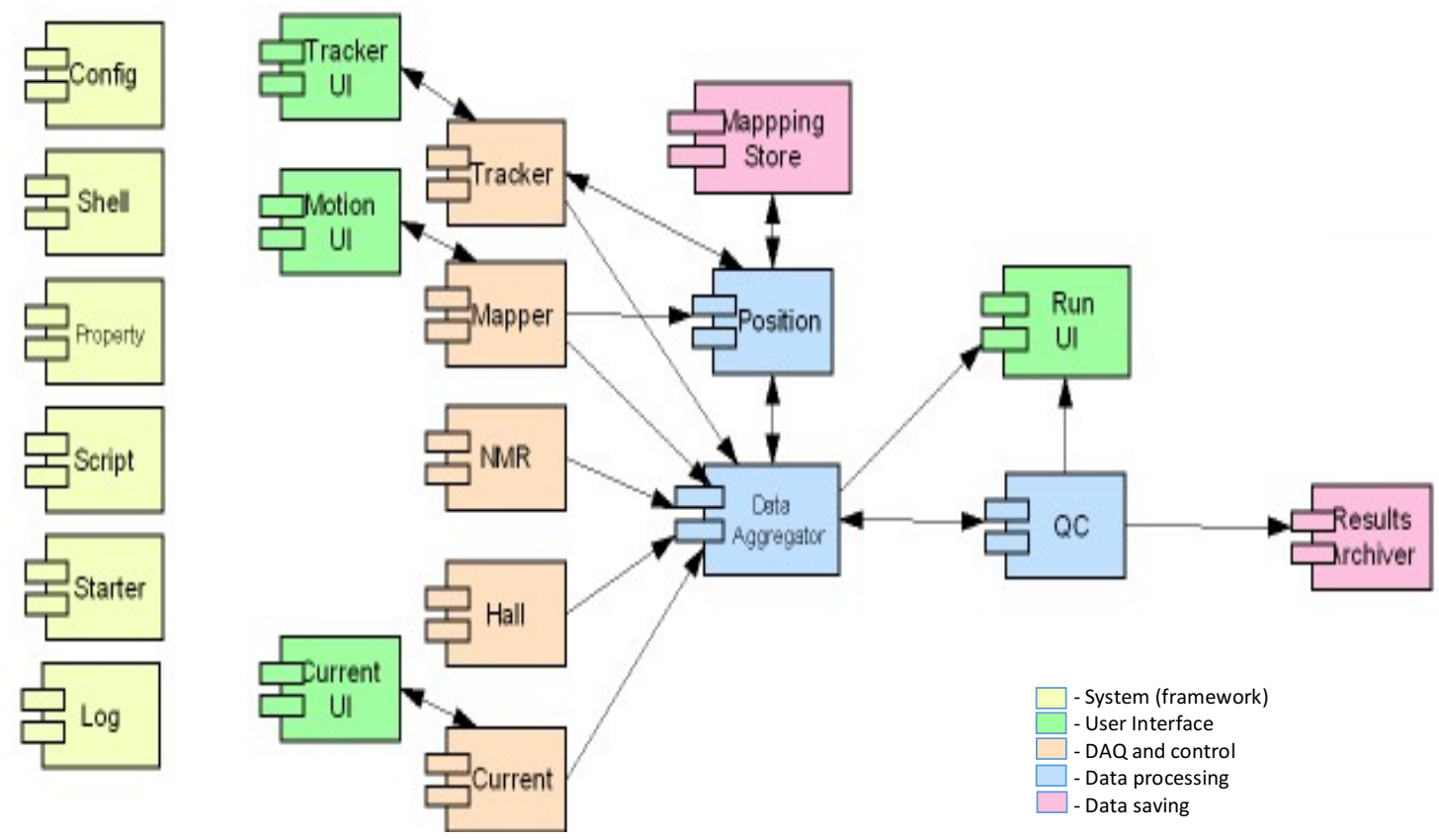

Fig. 4. FMS software components.

\section{Laser Tracker}

The Leica Absolute Laser Tracker AT403 is connected to the Mu2e FMS via a private network. This tracker has been selected due to its proven ability to work in a magnetic field of up to $200 \mathrm{G}$ with full functionality and accuracy [9]. The AT403 has a typical distance accuracy of $+/-0.005 \mathrm{~mm}$.

\section{E. Current}

The values of magnet current in the Mu2e magnets will be provided by the power supply control system via the EPICS channel access protocol.

\section{FMS SOFTWARE}

\section{A. Architecture}

The Mu2e FMS software is based on the EMMA framework [10], in which applications are constructed by the assembly of software components and specialized component functionality is selected via configuration information. An EMMA component is designed following the classical object model, where objects are separate entities with states and defined behavior and communicate with the system and other components via messages. The message-oriented middleware is implemented as a publish-subscribe software bus.

\section{B. Component Collaboration}

The FMS software is comprised of the system, DAQ and control, data processing, UI, and archival components (Fig. 4).
The components collaborate by exchanging data events, and their actions are orchestrated by a Python script [11]. Each command sent to a component is acknowledged by the Script component.

The measurement starts with the script reading its parameters that describe the measurement sections with different linear and angular steps. Next, all components are requested to initialize themselves, which involves obtaining their properties from the Property component and applying them. In the case of a DAQ component, it also includes an interaction with the hardware module.

The Config system component allows for choosing a system configuration, the script controlling the measurement, and script parameters specifying mapping regions and their granularity. The Starter component dynamically activates the components included in the configuration. Each activated component joins the software communication bus. All components respond to control events coming from the Script component by performing the requested operation (sent as a control event) and sending an acknowledgment. Significant events and exceptions are logged and displayed in the Shell UI component.

The script organizes the measurement in steps which correspond to readout operations by the Tracker, Hall, NMR and Current components. The data from these components are combined and restructured by the Aggregator, which sends its output to the Measurement UI and QC components. A measurement "sequence" contains all steps for a single axial position.

The QC (quality control) component performs data validation for each measurement step and sequence. The component checks whether the position coordinates given by the laser tracker and the field readouts given by Hall and NMR probes fall within the acceptable range of values. It also eliminates duplicate measurements and verifies that the number of suc- 
cessfully located target reflectors exceeds three on each "propeller", which allows for calculating 3D locations of the Hall probes. The QC component forwards measurement data to the Archiver component, which appends them to a TDMS file (a structured binary file format developed by National Instruments).

The Measurement UI provides visualization of the data and monitoring of the progress of the mapping process. During operation, the Position component caches data in the Mapping Store component.

\section{Laser Tracker Operations}

The system configuration contains two groups of target reflectors: a) the reference group, including stationary targets mounted on the Mu2e solenoids and possibly on the walls, and b) the measurement group, including targets mounted on the arms of the field mapper device. The measurement group targets are measured in each measurement step, and the reference group targets are measured at the beginning and end of each measurement sequence. The measurement of multiple targets is possible due to the ability of Leica AT403 to locate individual target reflectors without the need for continuous interferometric tracking.

Before the measurement is started under coordination of the script, the coordinates of all the targets must be introduced to the system so the system can automatically scan all stationary reflectors. It will then be able to calculate the approximate coordinates of the non-stationary reflectors based on their previous positions as well as the requested motion distance and rotation angle. To accomplish this, the mapper is positioned in its home position using manual motion and rotation controls provided by the UI. For each of the targets in the list, the operator selects one, manually points the laser tracker at it, waits for the lock signal and measures the target's coordinates. These coordinates are automatically recorded by the Mapping Store component. When all the target reflectors are measured, the system is ready for automatic, script-driven operations. This manual initialization must be repeated whenever the position of the laser tracker is changed.

The laser tracker operations are implemented by the Tracker and Position components working in tandem. The Tracker component implements access to the laser tracker whereas the Position component provides the list of optical targets (retroreflectors) to measure together with their predicted locations (coordinates). To measure a target, the Tracker component points the laser tracker at the target's predicted location, waits until the laser tracker locks onto the target and measures the target coordinates. The interactions with the laser tracker are implemented with the API library provided by the laser tracker manufacturer.

The measurement coordinated by the Script component, starts with the Tracker component initializing the laser tracker and measuring the targets from the reference and measurement groups with the field mapper in the home position. After each move of the mapper, the Position component calculates the predicted new positions of all non-stationary targets and checks, using a model of the mapper, if any target is hidden behind the mapper's column. Only the visible targets are attempted to be measured. The required laser tracker accuracy for measuring the large "propeller" is $0.07 \mathrm{~mm}$.

\section{User Interface}

The User Interface (UI) is implemented using a configurable component (Shell), which allows the embedding of the visual interface panels of other components to create a consolidated user interface. The plug-in components can show either their standard operation view or a debug view that permits inspection of the internal state of the component.

\section{1) UI Operation Panels}

There are five UI plugin components displaying data and status (Measurement) and manual control and monitoring of hardware (Mapper, Tracker, Position, and Current).

\section{2) UI Debug Panels}

An effective measurement process requires support in debugging problems encountered during the operation of the system. In the FMS, this is addressed by providing debugging panels for each component (with several selectable views). These panels allow the introspection of the internal state of the component, inspection of the input and output data, and the state and properties of the component and its associated hardware.

\section{Conclusion}

The magnetic flux density in the central part of the Mu2e detector, where the tracker and electromagnetic calorimeter are located, is required to be measured with a precision better than $1 \times 10^{-4}$. This limit is imposed by the precision tracking and momentum determination in the spectrometer region.

To address this need, a flexible field mapping system has been developed. What distinguishes this system from other detector field mapping systems using dedicated mappers (CMS, ATLAS) is the use of a laser tracker to concurrently track multiple targets on the mapper, which allows for accurate determination of the Hall probes' positions and orientations.

The Mu2e FMS fully automated and configurable component-based software is designed for rapid modification of measurement procedures and parameters. This system, with its built-in support for hardware and software observability and for debugging measurement problems, is especially suitable for long-term measurements with demanding timetables, such as the Mu2e detector field mapping.

\section{REFERENCES}

[1] R. H. Bernstein and P. S. Cooper, "Charged lepton flavor violation: An experimenter's guide," Jan. 2014. [Online]. Available: https://arxiv.org/abs/1307.5787, Accessed on: Nov. 29, 2021.

[2] Mu2e Collaboration, "Mu2e Technical Design Report", Mar. 2015. [Online]. Available: https://arxiv.org/abs/1501.05241, Accessed on: Nov. 29, 2021. 
[3] S. Feher et al., "Mu2e Solenoid Field Mapping System Design," IEEE Transactions on Applied Superconductivity, vol. 28, no. 3, pp. 1-5, Apr. 2018, Art no. 9001305, DOI: 10.1109/TASC.2017.2786720.

[4] V. I. Klyukhin et al., "Measurement of the CMS Magnetic Field," IEEE Transactions on Applied Superconductivity, VOL. 18, NO. 2, June 2008, 395-398.

[5] M. Alexa et al., Measurement of the ATLAS solenoid magnetic field, Journal of Instrumentation, Apr. 2008, DOI: 10.1088/17480221/3/04/P04003.

[6] F. Bergsma, H. Boterenbrood, BsCAN3, a Modular 3D Magnetic-Field Sensor System with CANopen Interface, CERN/Nikhef, Feb. 2013,

[Online]. Available: http://www.nikhef.nl/pub/departments/ct/po/html/Bsensor/BsCAN3.pdf, Accessed on: Nov. 2021.

[7] C. Orozco et al., "Hall Probe Calibration System Design for the Mu2e Solenoid Field Mapping System," IEEE Transactions on Applied Superconductivity (Volume: 28, Issue: 3, Apr. 2018), DOI: 10.1109/TASC.2018.2805830.

[8] PT2026 NMR Precision Teslameter, [Online]. Available: https://gmw.com/wp-content/uploads/2020/05/PT2026_BrochureA4_HD.pdf, Accessed on: Nov. 2021.

[9] A. Lippitsch et al., Leica Absolute Laser Tracker Operation in Magnetic Field Environment, Hexagon Manufacturing Intelligence, Unterentfelden, Switzerland.

[10] J. M. Nogiec and K. Trombly-Freytag, "EMMA: A new configurable software," in Proc. CHEP Conf., San Francisco, CA, USA, Oct. 2016.

[11] J. M. Nogiec et al., "The Application of Coordination to Magnetic Measurement Automation: An SSW System Example," IEEE Transactions on Applied Superconductivity, February 2020, PP(99):1-1 DOI:10.1109/TASC.2020.2974449. 